\title{
Dense stars with exotic configurations
}

\author{
W-Y. P. Hwang, C. Liu, and K. C. Tzeng \\ Department of Physics, National Taiwan University, Taipei, Taiwan 10764, Republic of China \\ Received August 15, 1990; revised version October 3, 1990
}

In this paper, we consider dense stars with configurations expected from the $S U(3)_{C} \times S U(2)_{W} \times U(1)$ standard model of strong and electroweak interactions. Following a recent suggestion that strange matter, a form of $(u d s)$ quark matter, may be the true ground state of hadronic matter, we investigate the prospect for the existence of dense stars consisting partially, or entirely, of strange matter by comparing the relative stability between neutron matter and strange matter. It is found that the restriction on the maximum star mass holds in all cases, including a pure strange star, a pure neutron star, and a neutron star with a quark core. It is also found that the choice of both the bag constant $B$ and the strong coupling constant $\alpha_{S}$ has a decisive effect on the relative stability between strange matter and neutron matter. For currently accepted values of $\left(B, \alpha_{S}\right)$, an $A=\infty$ dense star cannot consist entirely, nor partially, of strange matter. Nevertheless, such conclusion may be subject to change if corrections of $O\left(\alpha_{S}^{2}\right)$ or other effects are taken into account. Finally, we use the framework of Tolman, Oppenheimer, and Volkoff to analyze two cases of boson stars: gluon stars and stars consisting of massive scalar particles (massive bosons). It is found that, in the case of gluon stars, the presence of the bag constant in the QCD vacuum yields results very similar to that found in quark stars. On the other hand, soliton stars consisting of massive bosons exist if there is some background pressure which plays the role similar to the bag constant for lowering the matter pressure. The stability problem for both gluon stars and soliton stars is briefly discussed.

PACS: $21.65 .+\mathrm{f}$

\section{Introduction}

Over the last two decades, progresses in particle physics have been enormous so that the Glashow-Salam-Weinberg (GSW) theory [1] of electroweak interactions and quantum chromodynamics (QCD) [2] for describing strong interactions are now christened as the "standard model". In the standard model, the building blocks of all matter includes three generations of quarks and leptons, mediators of fundamental interactions $\left(\gamma, W^{ \pm}, Z^{0}\right.$, and gluons), and a Higgs particle (arising from the specific way how our space-time physical vacuum works). On the other front, nuclear physics has a very long history (more than 50 years) of success without a need to use quarks explicitly. The basic degrees of freedom in nuclear physics include mainly nucleons (protons or neutrons) and leptons such as $\left(e^{-}, v_{e}\right)$. Thus, in the context of nuclear physics, a stable stellar configuration, which consists of electrically neutral objects, may then vary from dwarfs (consisting primarily of hydrogenic atoms), to neutron stars (consisting primarily of neutrons), to black holes (gravitationally collapsed stellar objects). [3] With the advancement of the standard model in particle physics, it is obvious that we must re-examine the question of possible stellar corpses, using the basic degrees of freedom adopted in particle physics rather than in conventional nuclear physics.

One such important problem has to do with neutron stars. The inner part of a neutron star was originally supposed to be composed of neutron matter. However, there are a lot of neutrons being compressed in a small region, the neutrons may fuse with one another and becomes a huge cluster of deconfined quarks, called "quark matter". Two kinds of quark matter may be considered. One is $(u d)$ matter, which is a system of $u p$ and down quarks in chemical equilibrium. The other is strange matter [4], which is a system of up, down, and strange quarks in chemical equilibrium. Once it is energetically favorable for quark matter to have strange quarks in it (when the chemical potential or Fermi energy exceeds the strange quark mass), strange matter is always more stable than uid matter.

In earlier investigations [5], the question of whether a neutron star could have a quark core has been addressed by comparing the relative stability between neutron matter and quark matter. However, the quark core considered by these authors [5] refers to an (ud) matter one. As 
the $(u d)$ matter is less stable, it is sometime concluded [5] that an $A=\infty$ dense star cannot be a quark star at all. However, the central density of a neutron star may reach several times of the nuclear matter density, corresponding to a Fermi energy of the order of about $1 \mathrm{GeV}$, which is considerably higher than the strange quark mass adopted in quark models. Thus, if a neutron star has a core consisting of deconfined quarks, the possibility of having a strange-matter core, rather than a $(u d)$ matter one, must be considered. One of the main objectives of this investigation is to address this issue by comparing relative stability between neutron matter and strange matter. As we shall see, the prospect of having an $A=\infty$ dense star consisting entirely of strange matter, or partially of strange matter, depends very much on the choice of both the bag constant $B$ and the strong coupling constant $\alpha_{S}$, which has a decisive effect on the relative stability between strange matter and neutron matter. The objective of our study on strange stars also differs considerably from those in previous investigations [6,7], in which the stability of strange matter relative to neutron matter for realistic values of $\left(B, \alpha_{S}\right)$ was not seriously considered. Our investigation of this problem is described in some detail in Sects. II and III.

On the other hand, T.D. Lee and his co-workers [8] have recently suggested that soliton stars may exist as a new form of stable stellar configurations. According to them, a soliton star as heavy as $10^{12} M_{\odot}$ or a mini soliton star of the mass such as $10^{-3} M_{\odot}$, with $M_{\odot}$ the solar mass, may exist. In a related context, B.W. Lynn et al. [9] have considered $Q$-stars or $Q$-balls which have masses $\left(10^{-8}-10^{2}\right) M_{\odot}$ with corresponding radii $\left(10^{-8}-10^{3}\right)$ $\mathrm{km}$. By considering only couplings of gravitational strength, $\mathrm{Ph}$. Jetzer [10] has performed extensive analyses of the stability problem related to such boson stars [8]. As we shall see, the same stability problem can also be analyzed in the context of the Tolman-Oppenheimer-Volkoff (TOV) equation. Both analyses must be severely modified when couplings arising from interactions in the standard model are taken into account. Nevertheless, we investigate in Sect. IV stars consisting of boson matters, including gluons and massive scalar particles (massive bosons), using the TOV framework mentioned above.

\section{Relative stability between neutron matter and strange matter}

At a given value of pressure, the most stable phase of matter is the configuration with the least value of Gibb's free energy per baryon. Thus, the relative stability between neutron matter and strange matter can easily be determined, once we calculate the Gibb's free energy per baryon as a function of pressure for each configuration. For a proper description of quark matter [11], there are two important parameters, the bag constant $B$ and the strong coupling constant $\alpha_{S}$. The important role played by the strange quark mass has been emphasized previously by Farhi and Jaffe [11] but, as indicated earlier, a direct contrast with the case of neutron matter is yet to be done in order to decide if strange matter is relevant for the neutron star problem and in such context the bag constant $B$ and the strong coupling constant $\alpha_{S}$ play a direct and important role. Thus, the quark masses adopted in the present paper are taken from the best fit [12] (with $\left.\bar{m}=\left(m_{u}+m_{d}\right)=10 \mathrm{MeV}\right)$ to the observed baryon mass splittings in the context of a chiral bag model.

Specifically, we consider an electrically neutral system of quarks of possible flavors $u, d, s$, and $c$, in equilibrium with an electron gas. It is clear that the equilibrium may be achieved through the following weak reactions:

$$
\begin{gathered}
s+u \leftrightarrow d+u, \\
s+c \leftrightarrow d+c, \\
d+u \leftrightarrow d+c, \\
s+u \leftrightarrow s+c, \\
u+e^{-} \rightarrow d+v_{e}, \\
u+e^{-} \rightarrow s+v_{e}, \\
d \rightarrow u+e^{-}+v_{e}, \\
s \rightarrow u+e^{-}+\bar{v}_{e}, \\
c+e^{-} \rightarrow d+v_{e}, \\
c+e^{-} \rightarrow s+v_{e}, \\
d \rightarrow c+e^{-}+\bar{v}_{e}, \\
s \rightarrow c+e^{-}+\bar{v}_{e} .
\end{gathered}
$$

Note that neutrinos or antineutrinos produced in the equilibrium process may escape the system unless the temperature of the system is extremely high (such as $10^{110} \mathrm{~K}$ ) or its density is unusually high. Here we have in fact assumed that the system is sufficiently cold so that annihilation of antiquarks with quarks (into photons) already eliminates the antiquark content in the thermally equilibrated dense star (with $A=\infty$ ). Thus, we have, in view of (1),

$\varepsilon_{F}(u)=\varepsilon_{F}(c)=\varepsilon^{\prime}$,

$\varepsilon_{F}(d)=\varepsilon_{F}(s)=\varepsilon=\varepsilon^{\prime}+\varepsilon_{F}(e)$,

and

$2 n_{u}-n_{d}-n_{s}+2 n_{c}=3 n_{e}$.

The fermi energy for each species of fermions, which is just the chemical potential at $T=0$, is given by

$\varepsilon_{F}=\sqrt{p_{F}^{2} c^{2}+m^{2} c^{4}}$,

$p_{F}=h\left(\frac{3}{N_{c}} \pi^{2} n_{i}\right)^{1 / 3}$.

The energy density is given by

$\rho_{i}=\frac{N_{c}\left(m_{i} c^{2}\right)^{4}}{\pi^{2}(\hbar c)^{3}} f\left(x_{F}\right)$.

Here, (3) and (4), $N_{c}=3$ (the number of color) for each flavor of quarks and $N_{c}=1$ for electrons while the functions $f\left(x_{F}\right)$ is given by 


$$
\begin{aligned}
f\left(x_{F}\right) & \equiv \int_{0}^{x_{F}} x^{2} \sqrt{1+x^{2}} \mathrm{~d} x \\
& =\frac{1}{4} x_{F}\left(1+x_{F}^{2}\right)^{3 / 2}-\frac{1}{8} x_{F}\left(1+x_{F}^{2}\right)^{1 / 2} \\
& -\frac{1}{8} \ln \left\{x_{F}+\left(1+x_{F}^{2}\right)^{1 / 2}\right\}
\end{aligned}
$$

with $x_{F} \equiv p_{F} /\left(m_{i} c\right)$.

Thus, the baryon number density, the energy density, and the pressure for the entire system (at $T=0$ ) are given by

$n_{0}=\frac{1}{3}\left(n_{u}+n_{d}+n_{s}+n_{c}\right)$,

$\rho_{0}=\rho_{u}+\rho_{d}+\rho_{s}+\rho_{c}+\rho_{e}$,

$P_{0}=-\rho_{0}+P_{u}^{\prime}+P_{d}^{\prime}+P_{s}^{\prime}+P_{c}^{\prime}+P_{e}^{\prime}$,

with

$P_{i}^{\prime}=\frac{N_{c}\left(m_{i} c^{2}\right)^{4}}{3 \pi^{2}(\hbar c)^{3}} x_{F}^{3} \sqrt{1+x_{F}^{2}}$.

To take into account the fact that the QCD coupling $\alpha_{S}$ and the bag constant $B$ differ from zero, we use [11]

$n=n_{0}\left(1-\frac{2 \alpha_{S}}{\pi}\right)$,

$\rho=\rho_{0}\left(1-\frac{2 \alpha_{S}}{\pi}\right)+B$,

$P=P_{0}\left(1-\frac{2 \alpha_{S}}{\pi}\right)-B$

Since it is well-known that the QCD coupling $\alpha_{S}$ necessarily depends on the scale $Q^{2}$ (or the distance scale), we choose a simple ansatz in which $\alpha_{S}$ depends on the density as follows:

$$
\begin{aligned}
\alpha_{S}=\alpha_{S}(n) & =\bar{\alpha}_{S}, \quad \text { for } n<\bar{n}, \\
& =\frac{\tilde{\alpha}_{S}}{1+\tilde{\alpha}_{S} \ln \frac{n}{\bar{n}}}, \quad \text { for } n \geqq \bar{n},
\end{aligned}
$$

with $\bar{n}$ chosen somewhat arbitrarily to be $0.15 \mathrm{fm}^{-3}$ for the results reported in this paper. Note that (8) is very similar to what H. Stöcker [13] obtained previously. Our value of $\bar{n}$ corresponds to a QCD scale of slightly less than $200 \mathrm{MeV}$ which yields similar numerical results (in the range interesting to us) if we insert, in (8), a coefficient of $\approx 0.5$ in front of $\ln \frac{n}{n}$ but take a smaller value of $\approx 100 \mathrm{MeV}$ for the QCD scale. (Such replacement gives Stöcker's result for $T=0$.)

Finally, the Gibb's free energy per baryon, $G / N$, is then obtained:

$G / N=\frac{p+P}{n}$.
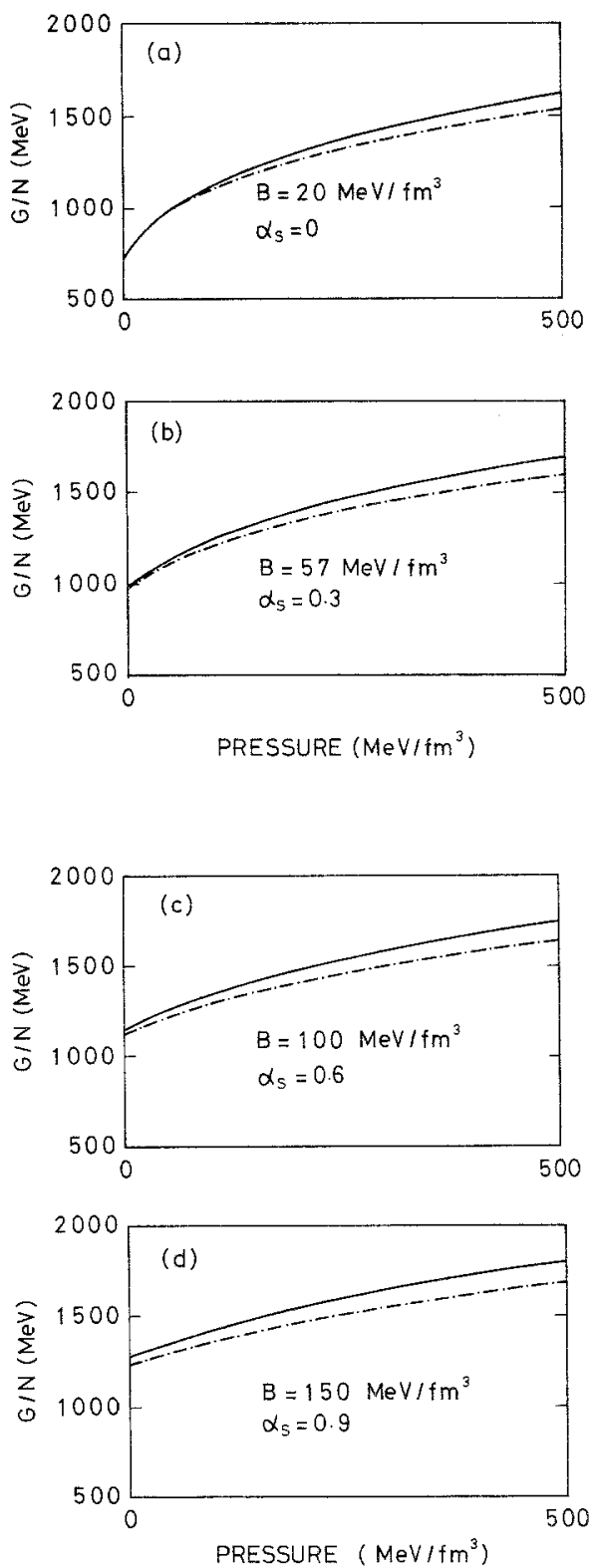

Fig. la-d. Gibb's free energy per baryon $(G / N)$ versus pressure for selected values of the bag constant $B$ and the strong coupling $\alpha_{S}$. Shown in solid curves are for $u d$ quark matter while in dash-dotted curves are for strange matter

Note that, to first order in $\alpha_{S}$, $G / N$ no longer depends on the strong coupling $\alpha_{S}$. Nor it depends on the bag constant $B$. However, the pressure $P$ depends explicitly on both $\alpha_{S}$ and $B$. As the relative stability of different phases of the matter is determined by comparing $G / N$ at the same $P$, with the phase of the smallest $G / N$ being the most stable one, the $G / N$ versus $P$ plot, as shown in Fig. 1 and 2 , depends on the parameters $\alpha_{S}$ and $B$ only through the dependence of $P$ on these two parameters.

In Figs. 1a-d, we plot the Gibb's free energy per baryon versus pressure of $(u d)$ matter and strange matter for various of $B$ and $\alpha_{S}$. It is seen that the Gibb's free energy of strange matter is always lower, except at the very low densities when the chemical potential is less than the strange quark mass (i.e. at the merging point of the 
two curves shown in Fig. 1a). Increase of the strange quark mass by a reasonable amount has the effect of moving the merging point of the two curves slightly toward the right side. Thus, in the range of the pressure which is relevant for the neutron star problem (approximately set by Figs. 1 and 2), the role played by the strange quark mass is relatively unimportant, as compared to the bag constant $B$ and the strong coupling constant $\alpha_{S}$.

Figures $1 \mathrm{a}-\mathrm{d}$ indicate that, except at the very low densities when the chemical potential is less than the strange quark mass, strange matter is always more stable than $(u d)$ matter for any $B$ and $\alpha_{S}$. In other words, $(u d)$ matter can lower its energy by changing the $u$ and $d$ quarks into strange and even charm ones through weak reactions listed in (1).

To compare the relative stability between neutron matter and strange matter, we use the phenomenological form obtained by Bethe and Johnson [14] in the description of neutron matter (which takes into account the shortrange repulsion between two neutrons). Thus, the energy density, the pressure, and the Gibb's free energy per baryon are specified, for neutron matter, by

$\rho=236 n^{2.54}+M_{n} n\left(\right.$ in $\left.\mathrm{MeV} / \mathrm{fm}^{3}\right)$,

$P=364 n^{2.54}\left(\right.$ in $\mathrm{MeV} / \mathrm{fm}^{3}$ ),

$G / N=\frac{\rho+P}{n}=600 n^{1.54}+M_{n}($ in $\mathrm{MeV})$,

with $M_{n}$ the neutron mass $(939.6 \mathrm{MeV})$.

We plot in Fig. 2(a) the Gibb's free energy per baryon versus pressure for neutron matter and strange matter with $\alpha_{S}=0$ and various values of $B$. Note that, at the very low densities when the chemical potential is less than the strange quark mass, the curve for strange matter is extended naturally by using that for the $(u d)$ matter.

Our numerical results indicate that, for $B<61 \mathrm{MeV} /$ $\mathrm{fm}^{3}$ and $\alpha_{S}=0$, strange matter is always more stable than neutron matter. For example, as in Fig. 2(a), the curve for strange matter is always below that for neutron matter when the value of $B$ is $57\left(\mathrm{MeV} / \mathrm{fm}^{3}\right)$. As the value of $B$ increases, the curve for strange matter moves upward and intersects the curve for neutron matter. This means that neutron matter now is more stable at lower densities, because its Gibb's free energy is lower. At the intersecting point which determines the critical pressure $P_{c r}$, neutron matter will convert to strange matter through a phase transition. Strange matter is still more stable at higher densities, because its Gibb's free energy is lower at high densities. In the jargon of the Maxwell construction, this means that, in the $P-n$ phase diagram, there is a region of $n$ at which the pressure is set equal to $P_{c r}$. Comparing the Gibb's free energy per baryon thus provides a simple way to determine the phase-transition point $P_{c r}$.

Where the intersecting point lies determines if formation of strange matter is relevant for the neutron star problem. Qualitatively speaking, there is a maximum central density for stable neutron stars and a neutron star with a central density higher than the maximum value will collapse upon a small perturbation. If the density at the intersecting point is higher than the maximum central
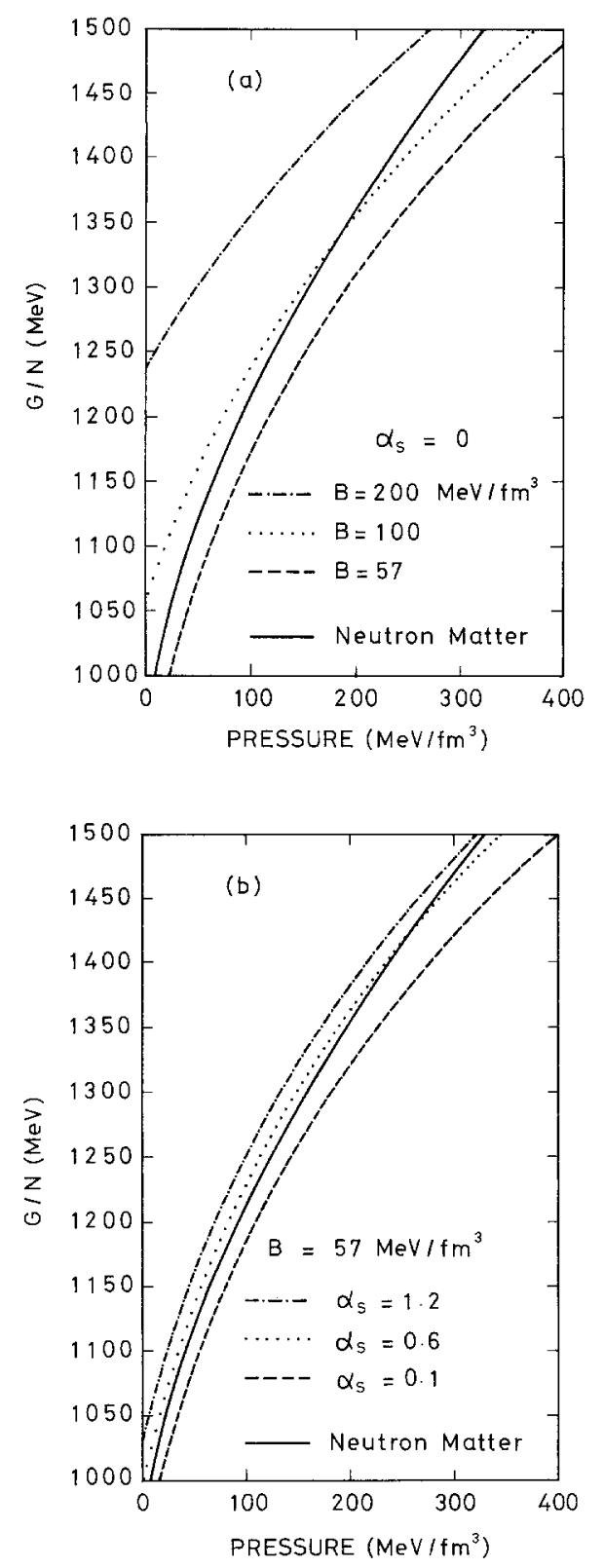

Fig. 2a, b. Gibb's free energy per baryon versus pressure for neutron matter (solid curve) and strange matter

density for stable neutron stars, then the issue concerning strange matter is irrelevant for the neutron star problem - since there will be no neutron stars with a strange matter core nor any strange star. In other words, strange matter is relevant for the neutron star problem if and only if the density at the intersecting point is lower than the maximum central density allowed for stable neutron stars since, then, there is a possibility for neutron stars having a strange matter core, or evcen for a pure strange star. This is why [5] we must contrast the quark matter result with the case of neutron matter. This critical point have not been fully appreciated so far in discussions $[6,7]$ of strange stars.

It is obvious that the larger the value of $B$ is, the higher the phase transition density is. On the other hand, if we fix the value of $B$, we get a very similar result as 
we vary the value of $\alpha_{S}$. Figure 2b shows the Gibb's free energy per baryon versus pressure for neutron matter and strange matter with $B=57\left(\mathrm{MeV} / \mathrm{fm}^{3}\right)$ and various values of $\alpha_{S}$. For small values of $\alpha_{S}$, strange matter is always more stable than neutron matter, because its Gibb's free energy is lower. For larger values of $\alpha_{S}$, neutron matter becomes more stable at lower densities; strange matter is more stable at higher densities; and there is a phase transition between neutron matter and strange matter at some critical point. Note that the larger the values of $\alpha_{S}$ is, the higher the phase transition density is. As mentioned earlier, the $G / N$ versus $P$ plot depends on $\alpha_{S}$ and $B$ through $P\left(\alpha_{S}, B\right)$ and, from (7c), we see that an increase on $\alpha_{S}$ (or $B$ ) reduces the pressure $P$ and moves the curve (with a positive slope) horizontally towards the left, making the system less stable.

Continuing on our discussion of the question if formation of strange matter is relevant at all for the neutron star problem, we see from Fig. 2a and b that "realistic values" for the bag constant $B$ and the strong coupling constant $\alpha_{S}$ in fact do not give us too much optimism. For example, the MIT bag model for $B\left(57 \mathrm{MeV} / \mathrm{fm}^{3}\right)$, which is often considered to be a little too low, allows for only $\alpha_{S}<1.2$ (which is already smaller than the MIT value [15] of 2.2) if formation of strange matter is relevant for the neutron star problem. It seems [16-18] that, to obtain reasonable hadron spectroscopy in bag models without pions, the values for $\alpha_{S}$ and $B$ are correlated such that a smaller $\alpha_{S}$ always comes with a larger $B$, or vice versa. It is clear, in existing models, [15-18] that, in order that strange matter is relevant in the context of neutron stars, one may already invoke the values of $B$ and $\alpha_{S}$ which are in fact unrealistically too low. Nevertheless, it may be hoped that the effect arising from the lowestorder QCD corrections might be dampened somewhat by higher order effects, but it remains to be seen if this is true.

\section{Strange matter and the neutron star problem}

We first mention very briefly the basics for stable spherical stars. It is well known that, in the standard stellar model for spherical stable dense stars, there are three basic equations:

(I) The equation of state (EOS) for the matter which forms the star

$$
P(r)=P(\rho(r))
$$

with $P(r)$ the pressure of the matter at the distance $r$ from the center of the star and $\rho(r)$ its energy density.

(2) The mass equation

$m(r)=\int_{0}^{r} 4 \pi r^{\prime 2} \rho\left(r^{\prime}\right) \mathrm{d} r^{\prime}$,

which states that the total mass inside a radius $r$ is equal to the integration of the mass density $\rho(r)$.

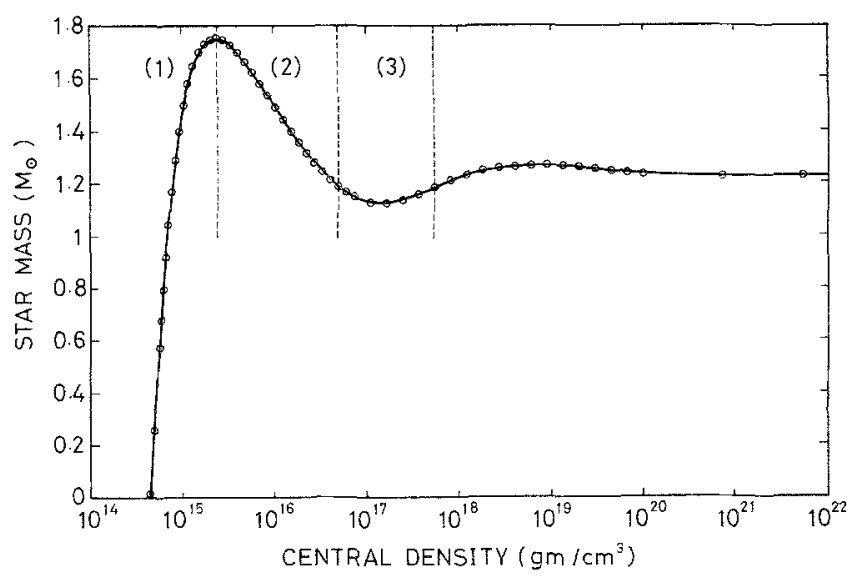

Fig. 3. The strange star mass versus its central density. Here we have used $B=57 \mathrm{MeV} / \mathrm{fm}^{3}$ and $\alpha_{S}=0$ which ensures existence of a strange star

(3) The Tolman-Oppenheimer-Volkoff (TOV) equation

$\frac{\mathrm{d} P}{\mathrm{~d} r}=-\frac{\left(m(r) c^{2}+4 \pi r^{3} P(r)\right)\left(\rho(r) c^{2}+P(r)\right)}{r\left(r c^{4} / G-2 m(r) c^{2}\right)}$,

which is derived from the Einstein equation with the Schwarzschild metric. It is essentially the statement that the inward gravitational force must be balanced by the outward pressure given by the EOS.

For a given central density of the star, we use the EOS to integrate numerically the mass equation and TOV equation outward until the star surface (where $P=0$ ) is reached. In this way, we get the star mass, the star radius, the density profile of the star, and so on.

As a sample result, we plot in Fig. 3 the star mass versus the central density for a star consisting of strange matter only. Here we have used $B=57 \mathrm{MeV} / \mathrm{fm}^{3}$ and $\alpha_{S}=0$ for the sake of illustration. The maximum point of the curve means that there is a restriction on the value of the star mass [19]. Like the case for a neutron star, the maximum star mass of a pure quark star is also restricted to only a couple of solar masses.

There is in fact the stability theorem [20] for stars consisting of a perfect fluid. The theorem tells precisely where the transition from stability to instability can occur. In our case, we just look for the critical central density $\rho_{c}$ such that $\mathrm{d} M / \mathrm{d} \rho$ vanishes at $\rho_{c}$ where $M$ is the star mass. As $\mathrm{d} M / \mathrm{d} N$ with $N$ the total particle number of the star usually differs from zero at $\rho_{c}$, one sees that $\mathrm{d} M / \mathrm{d} \rho=0$ implies $\mathrm{d} N / \mathrm{d} \rho=0$ as well. This can be done easily in the context of (11)-(13), i.e. the TOV framework. Otherwise, it is necessary [21] to apply small radial perturbations to the star configuration and derive the governing "pulsation equation". Using the stability theorem, we can determine the critical density $\rho_{c}$ at which the pulsation equation has a zero mode.

Therefore, the curve such as Fig. 3 is very useful. It can be divided into several regions, according to the positions of the values $\rho_{c}$ at which $\mathrm{d} M / \mathrm{d} p=0$. Stars in the first region, which extends from the threshold till the first $\rho_{c}$, are believed to be stable. As $\mathrm{d} M / \mathrm{d} r>0$, we see that, unless we add some mass to the star, it cannot shrink by 
itself and its central density will not increase spontaneously. The second region starts from the first $\rho_{c}$ to somewhere below the second $\rho_{c}$ (the minimum in the $M-\rho$ plot). A star lying in this region is unstable because, under a small perturbation, its inner part will shrink further and reach a higher density. Continuing in this way, the star may collapse indefinitely until perhaps it becomes a black hole. The existence of a third region, which centers around the second critical density $\rho_{c}$, is somewhat unexpected. It occurs at huge densities with $\rho \approx 10^{3} \rho_{\text {n.m. }}$, where $\rho_{\text {n.m. }}$. is the nuclear matter density. A star in this region cannot collapse indefinitely since it requires an additional mass in order to "climb" out of the valley centered around the second $\rho_{c}$ (the minimum point). We suspect that a dense star, if it so happens in reaching this region by, e.g., shedding its mass after $\rho$ passes the first critical point $\rho_{c}$, may oscillate in some way around the minimum point, providing a natural pulsating mechanism. The stability problem for stars lying in this region cannot be synthesized quantitatively without going through the tedious steps of deriving and solving the pulsation equation (which might be just impractical for a system containing several species of fermions interacting with the gravitational field).

Since the relative stability between neutron matter and strange matter is sensitive to the choice of $B$ and $\alpha_{S}$, a dense star may in principle be composed of neutron matter entirely, or of strange matter entirely, or of both of them. Once the values for $B$ and $\alpha_{S}$ are known, we can decide if strange matter is relevant for the question of $A=\infty$ dense stars.

In Fig. $4 \mathrm{a}$ we plot the star mass versus the central density of the star for $B=57 \mathrm{MeV} / \mathrm{fm}^{3}$ and various values of $\alpha_{S}$. For $\alpha_{S}<0.3$, we get a pure quark star, because the strange matter is more stable for these values of $B$ and $\alpha_{S}$. For $0.3<\alpha_{S}<1.2$, we have a neutron star with a quark core; its outer part is composed of neutron matter, and its inner part is composed of strange matter. The double vertical lines in Fig. $4 \mathrm{a}$ indicate the boundary of the small region of densities where neutron matter and quark matter coexist. For $\alpha_{S}>1.2$, the strange-matter core is irrelevant, because the phase transition from neutron matter to strange matter occurs at densities in the unstable region. In other words, the phase transition density is higher than the central density of ordinary neutron stars, so there is no quark core at all. For each case discussed above, the general feature of the curve is all the same and the restriction on the maximum star mass is always there.

In Fig. $4 b$ we fix the value of $\alpha_{S}$ as 0.3 , and the result is shown for several values of the bag constant $B$. For small values of $B\left(B<57 \mathrm{MeV} / \mathrm{fm}^{3}\right)$, we have a pure quark star. For intermediate values of $B(57 \mathrm{MeV} /$ $\left.\mathrm{fm}^{3}<B<150 \mathrm{MeV} / \mathrm{fm}^{3}\right)$, we get a neutron star with a quark core. And for large values of $B\left(B>150 \mathrm{MeV} / \mathrm{fm}^{3}\right)$, the quark-matter core is irrelevant. The restriction on the maximum star mass is also always there.

Note that, in the above discussions, we did not consider seriously the stars which happen to lie in the third (pulsating) region, primarily because it is difficult to imagine how a star can evolve to this stage at the first
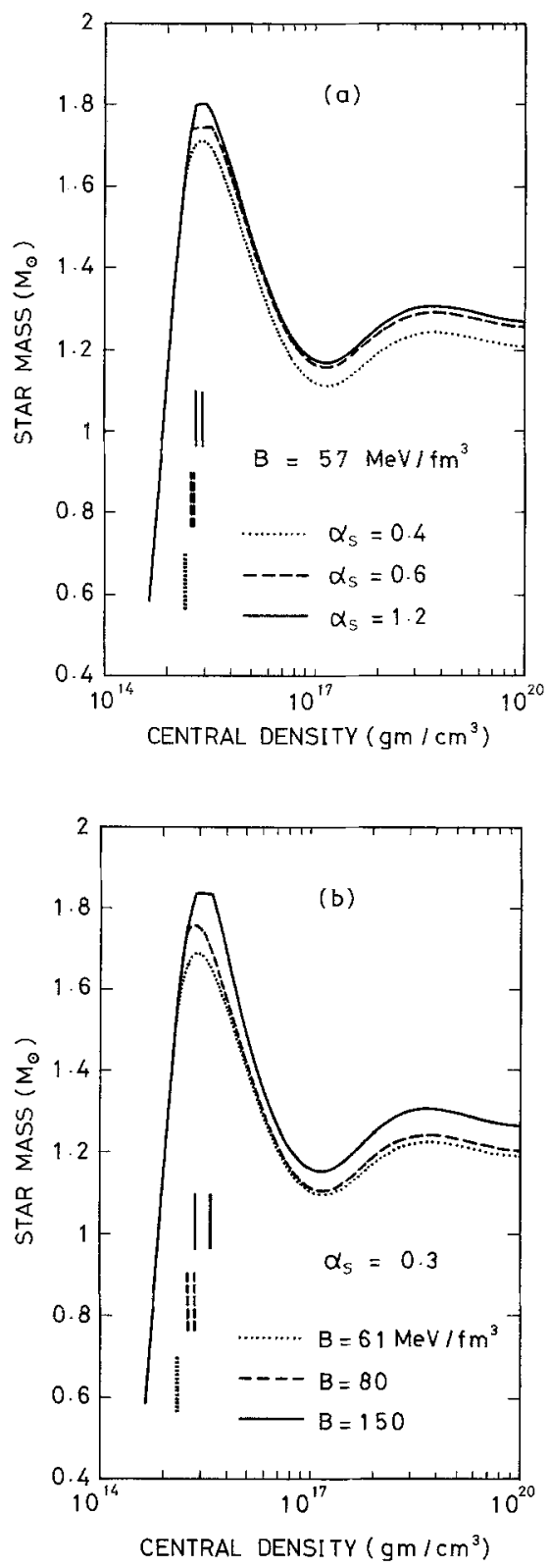

Fig. 4a, b. The star mass versus its central density for stars which consist of either neutron matter, or strange matter, or both, with the equation of state described in the text.

place. A heavy star many times the solar mass, if it does not explode before its central density passes the first critical $\rho_{c}$, will continue to collapse. Its central density will soon pass the phase transition point between neutron matter and strange matter, which is still well below the second critical $\rho_{c}$. Now, strange matter becomes relevant and the transition from neutron matter to strange matter, as caused by weak reactions listed in (1), may take place at a time scale much shorter than the characteristic time for gravitational collapse. This may result in a "supernovae explosion" but the scenario is completely different from what has been discussed in the literature $[6,7]$. In this new scenario which is undoubtedly rather speculative, the core may continue to collapse (if it is still too heavy) or pulsate in the third region. If this is still not 
speculative enough, we might mention that, after the first explosion due to transition into strange matter, there might soon be a second major calamity due to transition of strange matter into charmed strange matter. However, things may become completely irrelevant at some point if the event horizon separating the outside part from an inside black hole starts to appear.

To conclude this section, we wish to mention specifically that, for currently accepted values of $\left(B, \alpha_{S}\right)$, an $A=\infty$ dense star cannot consist entirely, nor partially, of strange matter. Nevertheless, such conclusion may be subject to change if corrections of $O\left(\alpha_{S}^{2}\right)$ or other effects are taken into account. The scenario concerning the third region is rather speculative and, if a star happens to reach this region, phase transition into strange matter will be of great importance.

\section{The case for boson stars}

Recently, T.D. Lee and his co-workers [8] have suggested that soliton stars may exist as a new form of stable stellar configurations. According to them, a soliton star as heavy as $10^{12} M_{\odot}$ or a mini soliton star of the mass such as $10^{-3} M_{\odot}$, with $M_{\odot}$ the solar mass, may exist. In a related context, B.W. Lynn et al. [9] have considered $Q$-stars or $Q$-balls which have masses $\left(10^{-8}-10^{2}\right) M_{\odot}$ with corresponding radii $\left(10^{-8}-10^{3}\right) \mathrm{km}$. By considering only couplings of gravitational strength, Ph. Jetzer [10] has performed extensive analyses of the stability problem related to such boson stars [8].

According to the stability theorem [20] which we have mentioned earlier in connection with the TOV equation, we can determine by using the star mass versus central density plot the critical density (or densities) at which the transition from stability to instability can occur. Instead of obtaining the pulsation equation [21] as Jetzer [10] did for soliton stars, we may in fact analyze the stability problem for boson stars using the TOV equation. This is particularly convenient when the star consists of multicomponents of matter. In all cases, however, we need to know the equation of state (EOS) for the bulk matter which the star consists of. In principle, we can derive the EOS using quantum statistical mechanics once the Hamiltonian is known. In practice, however, it can be done for only a few simple cases. Nevertheless, one may circumvent the difficulty by using only an approximate EOS, a practice that is often sufficient. Likewise, the pulsation equation can only be derived and solved for very simple cases.

The stability information obtained by using the TOV equation is of course far less than the pulsation equation as it does not contain any detailed dynamics. In both cases, however, the so-called "stability" makes sense only mechanically. For example, it is "stable" against radial excitations as in, e.g., Jetzer's studies of soliton stars. In practice, detailed dynamics related to strong and electroweak reactions must be present in order that the star be manufactured at the first place. In other words, if one assumes that complex scalar fields couple only to the gravitational field (or to other fields with couplings of gravitational strength), the star cannot be synthesized to begin with. If one allows for presence of strong and electroweak interactions, the star can be produced but $\mathrm{Yu}$ kawa couplings of scalar fields to matter (fermion) fields, or couplings of similar nature, must be present. One then expect that the conservation of the $Q$ charge (the number of the scalar particles), as advoacted in, e.g., studies of Lynn et al. [9] to stabilize the star, is violated severely (as compared to the gravitational effect). Therefore, we believe that, for boson stars of any kind, the stability cannot come from conservation of a global topology (or charge); instead, it must be associated with a local topology of some sort (if topology plays a role here at all).

The remarks which we have just made indicate that the suggestion for $Q$-stars or the stability analysis of Jetzer is still far from a complete story. Meanwhile, it is obviously useful to look into the mechanical stability problem for boson stars using the TOV equation. We shall briefly touch upon the more difficult, but realistic, stability question in each subsection. As there are only a very limited number of bosons in the standard model, the potentially interesting list is rather short: (1) gluon stars, or (2) stars consisting of massive bosons such as Higgs particles.

\section{(1) Gluon stars}

Gluon matter with trivial local structure probably cannot exist for very long in the time scale typical for stellar evolution. However, the nonabelian feature of QCD offers ways to construct solutions of topological nature, just like the Skryme solutions in the case of $S U(2)$. The gluon matter consists of numerous such topologoical nuggets will of course be very stable. On the other hand, it is quite likely that gluon matter consisting of numerous glueballs (which are not topological in nature), rather than a simple deconfined phase, is the most realistic picture for gluon matter. Unlike gluon matter consisting of topological gluon nuggets, such gluon matter can easily be manufactured during the early universe. Note that glueballs with unusual quantum numbers can be longlived because of the absence of decay channels. The scenario is then that, during the early universe, gluon stars of the various kinds get produced but, for stars without intrinsical topology, trivial gluon contents will soon be eliminated, through either decays or reactions, from the stars, yielding residual stars which are relatively longlived or even stable.

As a first approximation, we may consider the gluon matter in a residual gluon star as a free boson gas in a giant MIT bag. Thus we may integrate the TOV and mass equations outward until $P=B$ with $B$ the bag constant. For the sake of illustration, we shall use the MIT value, $B=57 \mathrm{MeV} / \mathrm{fm}^{3}$. Alternatively, we take

$P=P_{0}-B, \quad \rho=\rho_{0}+B, \quad P_{0}=\frac{1}{3} \rho_{0}$,

which treat gluon matter as a system of non-interacting massless Bose gas. Equation (17) yields an EOS:

$P=\frac{1}{3}(\rho-4 B)$, 


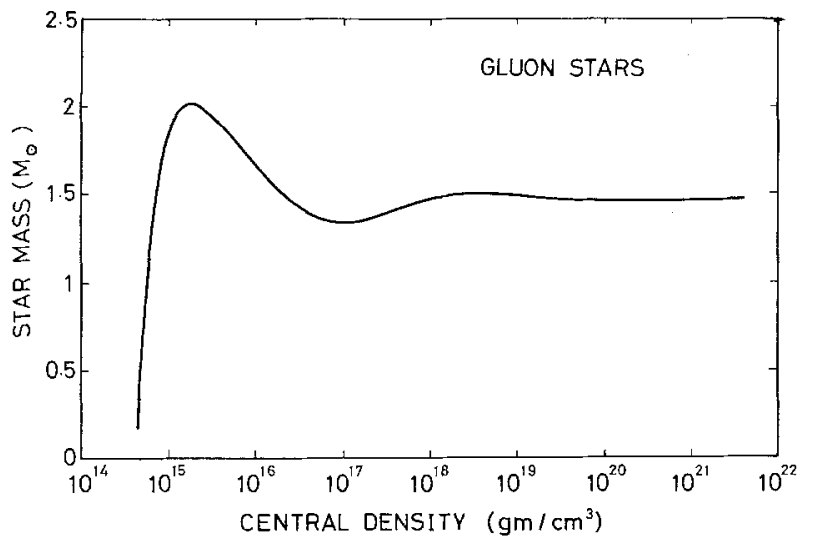

Fig. 5. The gluon star mass verus its central density. Here $B=57 \mathrm{MeV} / \mathrm{fm}^{3}$ (MIT bag model value) is used

which is identical to a system of non-interacting massless quark gas.

A typical result for gluon stars is shown in Fig. 5, which is of course almost identical with that of quark stars shown earlier. There is a maximum star mass in the case of gluon stars, $M_{\max }=2 M_{\odot}$. The corresponding critical density $\rho_{c}$ is where the transition from stability to instability takes place.

The questions concerning leading QCD corrections to gluon matter, possible stable forms of gluon matter, and dynamical evolution of related gluon stars are yet to be investigated.

\section{(2) Stars consisting of massive bosons}

In the standard model, the Higgs particle is now known [22] to be very heavy, i.e., greater than $41.6 \mathrm{GeV}$. It is unlikely that a star consisting of numerous such Higgs, even if produced during the early universe, can live for very long since such standard model Higgs will decay quickly into a heavy quark-antiquark pair. The electroweak coupling to the matter field, as needed to synthesize the star to begin with, annihilates the matter content locally, thereby making the star very short-lived. In order that suggestions of T.D. Lee and his co-workers [8] be relevant in astrophysics, one must either think of standard model Higgs solutions with nontrivial local topology or, by going beyond the standard model, work with a scalar field which, when cold (at small $Q^{2}$ ), interacts feebly with the matter field while, when hot (at large $Q^{2}$ ), interacts strongly with the matter field. This may also be considered as a sufficient criterion for saving the idea [9] of $Q$-stars or $Q$-balls.

Let us now use the TOV framework to analyze the stability problem for such boson stars. We may consider boson stars which have cooled to the extent that the temperature is much lower than the boson mass. In addition, the volume of the star is infinite as far as statistical mechanics is concerned. Thus, we may treat, as a zeroth order approximation, stars consisting of massive bosons as a system of a non-relativistic boson gas and use the
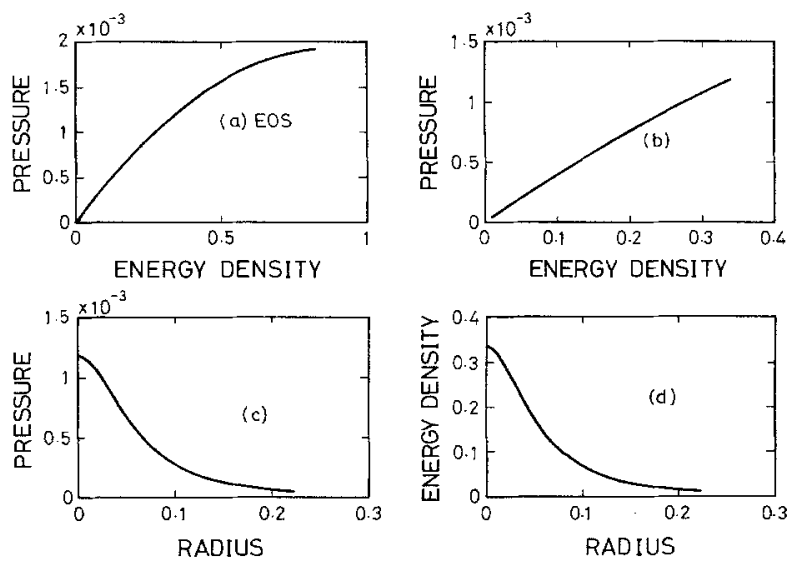

Fig. 6a-d. The EOS for a typical massive-boson gas (a) and the profile of the corresponding star (b-d). The pressure and energy density are in units of $M_{\odot} / \mathrm{km}^{3}$. The radius, i.e. the distance from the star center, is in units of kilometers

grand canonical ensemble with the volume $V \rightarrow \infty$ in order to obtain the EOS. For the sake of simplicity, we shall first neglect the complication due to possible BoseEinstein condensation. In addition, we neglect the effect caused by the temperature gradient of the macroscopic system. Using the formulae in the Appendix, we find that the EOS is given by

$$
\begin{aligned}
P & =\frac{2}{3} \rho_{0} \\
& =\frac{2}{3}\left(\rho-m c^{2} \cdot n\right),
\end{aligned}
$$

where $\rho_{0}$ is the kinetic energy density in the non-relativistic limit; $P, n$, and $\rho$ are the pressure, the number density, and the total energy density of the system, respectively; and $m$ is the mass of each individual particle.

In Fig. 6a, we plot the EOS for a typical massiveboson gas with temperature $T=10^{12}{ }^{\circ} \mathrm{K}$ and particle mass $m=20 \mathrm{GeV}$. The profile of a corresponding star is illustrated by Figs. 6b-6d. Of course, it is well known that the direct proportionality of the pressure against the total energy density gives rise to the result that, for an arbitrary central density, it is not possibie to integrate outward until $P=0$ (which defines the star surface). However, one may imagine that there exists a background pressure which plays the role of the bag constant in the case of gluon stars. In other words, massive bosons in question can exist only in a region with the energy density, i.e. some "background pressure", higher than that in true vacuum by a certain amount. Note that there are models which has such property. Among them, the nontopological soliton model proposed by T.D. Lee [8] predicts massive $\sigma$ bosons which exist in the false vacuum. (To say it more precisely, the $\sigma$ boson will choose to live in the false vacuum if its mass in the false vacuum is considerably less than the corresponding mass in true vacuum - by construction, of course.) Presumably, QCD has such property, yielding gluon stars as one of its consequences.

As an illustrative example, we choose $P_{B}=57 \mathrm{MeV} /$ $\mathrm{fm}^{3}$ (MIT bag constant) together with a boson mass of 


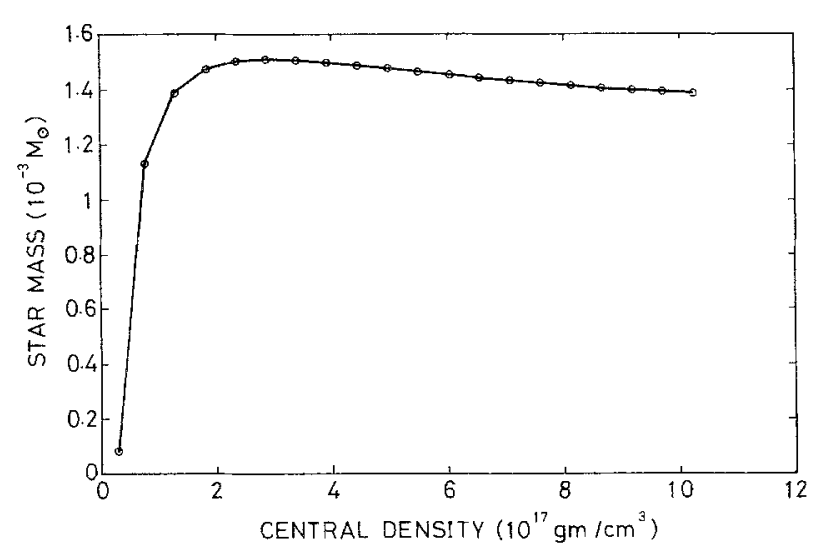

Fig. 7. The massive-boson star mass versus its central density. Here the input temperature, background pressure, and the mass of the scalar particle are $T=10^{12}{ }^{\circ} \mathrm{K}, P_{B}=57 \mathrm{MeV} / \mathrm{fm}^{3}$, and $m=20 \mathrm{GeV}$, respectively

$20 \mathrm{GeV}$ which yields the maximal star mass which is about $1.5 \times 10^{-3} M_{\odot}$ with corresponding $\rho_{c}=2.5 \times 10^{17} \mathrm{gm} / \mathrm{cm}^{3}$. The result is illustrated in Fig. 7. The existence of the critical density which separates unstable stars from stable ones suggests the possible existence for stable boson stars, as one may also infer rigorously from analyses $[8,10]$ of the pulsation equation. Here one notes that the analysis using the TOV equation may also be made rigorous if the EOS can be derived directly from the underlying lagrangian. Although one might associate the stars shown in Fig. 7 with possible mini soliton stars $[8,9]$, such connection must be made with great care since it is difficult to relate our choice of the EOS and $P_{B}$ (which is only a crude approximation) to the bulk matter property of the mini soliton stars (which is left untouched so far in their approaches $[8,9,10])$.

In the example shown above, inclusion of Bose-Einstein condensation affects very little since, for temperature $T=10^{12}{ }^{\circ} \mathrm{K}$ and particle mass $m=20 \mathrm{GeV}$ as adopted in the figures, the corresponding condensation energy density is about $2 \times 10^{18} \mathrm{gm} / \mathrm{cm}^{3}$ which is much higher than the densities relevant for Figs. 6 and 7. Nevertheless, the detailed dynamics as the temperature of soliton stars cools down presents an interesting but difficult problem which requires further studies.

It is clear that the input parameters, the mass of the scalar field and the background pressure (or the energy of the false vacuum), govern what kind of boson stars which one might get. It is relatively easy to cook up models which give rise to boson stars of desirable size and mass but, as already mentioned earlier, it is nontrivial to couple the scalar field(s) to the matter fields such that stars can be produced during the early universe but do not die out too quickly.

\section{Summary}

In this paper, we have considered dense stars with configurations expected from the $S U(3)_{C} \times S U(2)_{W} \times U(1)$ standard model of strong and electroweak interactions.
Our primary emphasis has to do with the question of whether strange matter is relevant for the neutron star problem. Following a recent suggestion [4] that strange matter, a form of ( $u d s)$ quark matter, may be the true ground state of hadronic matter, we have investigated in Sects. II and III the prospect for the existence of dense stars consisting partially, or entirely, of strange matter by comparing the relative stability between neutron matter and strange matter. It is found that the restriction on the maximum star mass holds in all cases, including a pure strange star, a pure neutron star, and a neutron star with a quark core. It is also found that the choice of both the bag constant $B$ and the strong coupling constant $\alpha_{S}$ has a decisive effect on the relative stability between strange matter and neutron matter. For currently accepted values of $\left(B, \alpha_{S}\right)$, an $A=\infty$ dense star cannot consist entirely, nor partially, of strange matter. Nevertheless, such conclusion may be subject to change if corrections of $O\left(\alpha_{S}^{2}\right)$ or other effects are taken into account.

In Sect. IV, we have used the framework of Tolman, Oppenheimer, and Volkoff to analyze two cases of boson stars: gluon stars and stars consisting of massive scalar particles (massive bosons). It is found that, in the case of gluon stars, the presence of the bag constant in the QCD vacuum yields results very similar to that found in quark stars. On the other hand, soliton stars consisting of massive bosons exist if there is some background pressure which plays the role similar to the bag constant for lowering the matter pressure. The stability problem for both gluon stars and soliton stars was briefly discussed.

This work is supported in part by the National Science Council of the Republic of China. One of us (W-Y. P. Hwang) wishes to acknowledge S. E. Koonin at Caltech and B. D. Keister at CarnegieMellon University for helpful discussions on the subject. He also wishes to acknowledge the Humboldt Foundation for the research fellowship, during which the revised manuscript was completed.

\section{Appendix}

In this Appendix, we review briefly the equation of state (EOS) adopted in Sect. IV.

We use the grand canonical ensemble [23] to determine the EOS. First, we recall a few basic formulae in the grand canonical ensemble: Let $Z(z, V, T)$ be the grand partition function as a function of the fugacity $z$, the volume $V$, and the temperature $T$. Let $\beta \equiv \frac{1}{k_{B} T}$.

\section{Equation of state:}

$$
\begin{aligned}
\beta P V & =\ln Z, \\
N & =z \frac{\partial}{\partial z} \ln Z, \\
U & =-\frac{\partial}{\partial \beta} \ln Z .
\end{aligned}
$$

For a system of free Bose gas, we have 


$$
\begin{aligned}
\ln Z & =\sum_{\vec{p}} g_{S} \ln \frac{1}{1-z \mathrm{e}^{-\beta \varepsilon p}} \\
& \stackrel{V \rightarrow \infty}{\longrightarrow} \frac{V}{h^{3}} g_{S} \int_{0}^{\infty} 4 \pi p^{2} \mathrm{~d} p \ln \frac{1}{1-z \mathrm{e}^{-\beta \varepsilon}},
\end{aligned}
$$

with $g_{S}$ the degeneracy factor. We note that effects caused by Bose-Einstein condensation can be neglected in the limit $V \rightarrow \infty$.

(1) massless case: $\varepsilon=p c$

$P=g_{S} \frac{8 \pi}{(h c)^{3}} g_{4}(z) \beta^{-4}$,

$\rho=\frac{U}{V}=3 g_{S} \frac{8 \pi}{(h c)^{3}} g_{4}(z) \beta^{-4}$.

Thus, $P=\frac{\rho}{3}$ comes out in a simple manner.

(2) non-relativistic massive case: $\varepsilon_{0}=\frac{p^{2}}{2 m}$

$$
P=\left(\frac{2 \pi m}{h^{2}}\right)^{3 / 2} g_{5 / 2}(z) \beta^{-5 / 2}
$$

$\rho_{0}=\frac{3}{2} P, \quad n=\left(\frac{2 \pi m}{h^{2}}\right)^{3 / 2} g_{3 / 2}(z) \beta^{-3 / 2}$,

where $g_{n}(z)=\sum_{j=1}^{\infty} \frac{z^{j}}{j^{n}}$.

\section{References}

1. Weinberg, S.: Phys. Rev. Lett. 19, 1264 (1967); Salam, A.: In: Elementary particle physics (Nobel Symposium No. 8). Svartholm, N. (ed.), p. 367. Stockholm: Almquist and Wiksell 1968; Glashow, S.L.: Nucl. Phys. 22, 519 (1961)

2. See, e.g., Muller, A.H.: Phys. Rep. 73C, 237 (1981); Reya, E.: Phys. Rep. 69C, 195 (1981)

3. Shapiro, S.L., Teukolsky, S.A.: Black holes, white dwarfs, and neutron stars. New York: John Wiley \& Sons 1983
4. Witten, E.: Phys. Rev. D30, 272 (1984)

5. Baym, G., Chin, S.A.: Phys. Lett. 62 B, 241 (1976); Keister, B.D., Kisslinger, L.S.: Phys. Lett. 64B, 117 (1976)

6. Alcock, A., Farhi, E., Olinto, A.: Astrophys. J. 310, 261 (1986); Haensel, P.: Prog. Theo. Phys. Supp. 91, 268 (1987)

7. Benvenuto, O.G., Horvath, J.E.: Phys. Rev. Lett. 63, 716 (1989); Horvath, J.E., Benvenuto, O.G.: Phys. Lett. B213, 516 (1988)

8. Lee, T.D.: Comments Nucl. Part. Phys. 17, 225 (1987); Lee, T.D.: Phys. Rev. D35, 3637 (1987); Friedberg, R., Lee, T.D., Pang, Y.: Phys. Rev. D35, 3640, 3658 (1987); Lee, T.D., Pang, Y.: Nucl. Phys. B315, 477 (1989)

9. Lynn, B.W.: Nucl. Phys. B321, 465 (1989); Bahcall, S., Lynn, B.W., Selipsky, S.B.: Nucl. Phys. B325, 606 (1989); and B331, $67(1990)$

10. Jetzer, Ph., Bij, J.J. van der: Phys. Lett. B227, 341 (1989); Jetzer, Ph.: Nucl. Phys. B316, 411 (1989); Jetzer, Ph.: Phys. Lett. B222, 447 (1989); B231, 433 (1989); B243, 36 (1990)

11. Freedman, B.A., McLerran, L.D.: Phys. Rev. D16, 1130 (1977); Farhi, E., Jaffe, R.L.: Phys. Rev. D30, 2379 (1984)

12. Hwang, W-Y.P.: Phys. Rev. D31, 2826 (1985); Hwang, W-Y.P., Lichtenberg, D.B.: Phys. Rev. D35, 3526 (1987)

13. Stöcker, H.: Nucl. Phys. A418, 587c (1984)

14. Bethe, H.A., Johnson, M.B.: Nucl. Phys. A230, 1 (1974)

15. DeGrand, T.A., Jaffe, R.L., Johnson, K., Kiskis, J.: Phys. Rev. D12, 2060 (1975)

16. Bartelski, J., Szymacha, A., Ryzak, Z., Mankiewicz, L., Tatur, S.: Nucl. Phys. A424, 484 (1984)

17. Chanowitz, M., Sharpe, S.: Nucl. Phys. B222, 211 (1983)

18. Carlson, C.E., Hansson, T.H., Peterson, C.: Phys. Rev. D27, 1556 (1983)

19. Rhoades Jr., C.E., Ruffini, R.: Phys. Rev. Lett. 32, 324 (1974)

20. Harrison, B.K., Thorne, K.S., Wakano, M., Wheeler, J.A.: Gravitation theory and gravitational collapse, Appendix B. (Chicago: University of Chicago Press 1965.) Weinberg, S.: Gravitation and cosmology, p. 305. New York: Wiley 1972; Straumann, N.: General relativity and relativistic astrophysics, p. 290. Berlin, Heidelberg, New York: Springer 1984

21. Chandrasekhar, S.: Phys. Rev. Lett. 12, 114, 437 (1964); Astrophys. J. 140, 417 (1964)

22. Decamp., D., et al., ALEPH Collaboration: Phys. Lett. B236, 233 (1990); B241, 141 (1990); B245, 289 (1990); B246, 306 (1990); Abreu, P., et al.; DELPHI Collaboration: Nucl. Phys. B342, 1 (1990); Adeva, B., et al., L3 Collaboration: L3 preprint \# 10, Phys. Lett. B (to be published); Akrawy, M., et al., OPAL Collaboration: Phys. Lett. B236, 224 (1990)

23. Huang, K.: Statitical mechanics. New York: John Wiley \& Sons 1987 